\title{
Effects of aerogel-like disorder on the critical behavior of $O(m)$-vector models. Recent simulations and experimental evidence *
}

\author{
C.Vásquez ${ }^{1}$, R.Paredes ${ }^{2,3}$ \\ ${ }^{1}$ Departamento de Física, Universidad Simón Bolívar, \\ Apartado 89000, Caracas 1080A, Venezuela \\ 2 Centro de Física, Instituto Venezolano de Investigaciones Científicas, \\ Apartado 21827, Caracas 1020A, Venezuela \\ 3 Particle Technology Group, Delft University of Technology, \\ Jualianalaan 136, 2628 BL Delft, The Netherlands
}

Received April 10, 2006, in final form May 12, 2006

\begin{abstract}
We review recent results on the effect of a specific type of quenched disorder on well known $O(m)$-vector models in three dimensions: the $X Y$ model (3DXY, $m=2$ ) and the Ising model (3DIS, $m=1$ ). Evidence of changes of criticality in both systems, when confined in aerogel pores, is briefly referenced. The 3DXY model represents the universality class to which the $\lambda$-transition of bulk superfluid ${ }^{4} \mathrm{He}$ belongs. Experiments report interesting changes of critical exponents for this transition, when superfluid ${ }^{4} \mathrm{He}$ is confined in aerogels. Numerical evidence has also been presented that the 3DXY model, confined in aerogel-like structures, exhibits critical exponents different from those of bulk, in agreement with experiments. Both results seem to contradict Harris criterion: being the specific heat exponent negative for the pure system $\left(\alpha_{3 \mathrm{DXY}} \simeq-0.011<0\right)$, changes should be explained in terms of the extended criterion due to Weinrib and Halperin, which requires disorder to be long-range correlated (LRC) at all scales. In numerical works, aerogels are simulated by the diffusion limited cluster-cluster aggregation (DLCA) algorithm, known to mimic the geometric features of aerogels. These objects, real or simulated, are fractal through some decades only, and present crossovers to homogeneous regimes at finite scales, so the violation to Harris criterion persists. The apparent violation has been explained in terms of hidden LRC subsets within aerogels [Phys. Rev. Lett., 2003, 90, 170602]. On the other hand, experiments on the liquid-vapor (LV) transition of ${ }^{4} \mathrm{He}$ and $\mathrm{N}_{2}$ confined in aerogels, also showed changes in critical-point exponents. Being the LV critical-point in the $O(1)$ universality class, criticality may be affected by both short-range correlated (SRC) and LRC subsets of disorder. Simulations of the 3DIS in DLCA aerogels can corroborate experimental results. Both experiments and simulations suggest a shift in critical exponents to values closer to the SRC instead of those of the LRC fixed point.
\end{abstract}

Key words: phase transitions, vector models, correlated disorder, aerogels

PACS: $64.60 . \mathrm{Cn}, 64.60 . \mathrm{Fr}, 64.70 .-p$

\section{Harris criterion in brief: original and extended}

Whether the presence of disordered impurities affects the critical behavior of an ideal system, or not, has been the task of numerous works through years. Since Harris' seminal work [1], a robust theoretical background has emerged to establish conditions for the relevance of disorder to phase transitions, which concern the criticality of the original pure system as well as the geometrical features of the disordered distribution of defects [2-5]. On the other hand, many experimental contributions in the last two decades posed interesting questions about criteria of relevance, supplying results that challenge predictions made by previous theoretical works. Amongst all, those on the superfluid transition of ${ }^{4} \mathrm{He}$ in light aerogels are rather intriguing [6-11].

Changes of critical exponents for the so called $\lambda$-transition, when superfluid ${ }^{4} \mathrm{He}$ is confined in aerogel pores, were reported repeatedly from late 80 's through late 90 's, with almost the same

\footnotetext{
${ }^{*}$ Contribution to the Mochima theoretical physics spring school. Joint CEA-IVIC-SFP workshop on Foundations
} of Statistical and Mesoscopic Physics. 2005 - World Year of Physics. 
question left open: do these results violate Harris and/or other relevance criteria? The question arises because aerogels are homogeneous (non-correlated) beyond a finite scale [12,13], and given that the specific heat exponent $(\alpha)$ is negative for this transition, exponents should not change after Harris criterion. Many numerical and theoretical works have since emerged to explain these contradictory results $[4,5,14-17]$.

According to Harris criterion [1], short-range correlated (SRC) disorder is irrelevant for the critical behaviour of any $d$-dimensional pure system which undergoes a second order phase transition with a correlation length exponent $\nu_{\text {pure }}>2 / d$. The criterion was shown valid if disorder presents a correlation function $\delta(\overrightarrow{\boldsymbol{r}})$ and, after Josephson hyperscaling [18], $\alpha_{\text {pure }}<0$. This original work was improved several years later by Weinrib and Halperin (WH) [2], who established a more general criterion of relevance: even if $\alpha<0$ for the pure system, it will change critical exponents if disorder is "correlated enough". A proper definition of this long-range correlated (LRC) disorder makes use of the impurity-impurity correlation function $g(r)=\langle n(r) n(0)\rangle$ and its long-range scaling exponent $\left(g(r) \sim r^{-a}, r \rightarrow \infty\right)$. Depending on how fast the tail of $g(r)$ decays, the extended criterion reads as follows:

$$
\begin{aligned}
& 2-d \nu_{\text {pure }} \geqslant 0 \quad \text { for } \quad a \geqslant d, \\
& 2-a \nu_{\text {pure }}>0 \quad \text { for } \quad a<d .
\end{aligned}
$$

In both cases disorder is relevant. Case (1) extends the particular definition of short-range correlated (SRC) disorder, made by Harris, to disorder distributions with a "fast-decaying" tail $(a>d)$. The second case $(2)$ is valid even if $\nu_{\text {pure }}>2 / d$. Based on one-loop expansions in $\epsilon=4-d \ll 1$ and $\delta=4-a \ll 1$, WH also argued that the correlation length exponent would be $\nu_{\mathrm{LRC}}=2 / a$, independent of the internal dimension $(m)$ of the order parameter. Prudnikov et al. [5] performed more accurate estimates to deduce $m$-dependent corrections, i.e., under the same kind of LRC distribution of defects (same $a$ ), their two-loop field theoretical expansions give different critical exponents for different $O(m)$ systems. Figure 1 graphically resumes what they obtain for the correlation length exponent $\nu_{\mathrm{LRC}}$, at disorder correlation exponents $2<a<3$ and $m=1,2,3$, quite different from the $m$-independent plot predicted by WH [2].

\section{Phase transitions in aerogels: experiments}

In this section, we review some important experimental results about critical systems confined in aerogels. In 1988, Moses Chan et al. [6] reported on the effect of quenched disorder on the superfluid (SF) transition of confined ${ }^{4} \mathrm{He}$, using three types of porous glass: Vycor, aerogels and xerogels. They measured the temperature dependence of the relative superfluid density, which scales as $\rho_{s} / \rho \sim t^{\zeta}$ near the critical point, being $t=\left|T-T_{\mathrm{c}}\right| / T_{\mathrm{c}}$ the reduced temperature, and the exponent $\zeta \equiv \nu$ due to hyperuniversality $[9,11]$. In the first case $\left({ }^{4} \mathrm{He}-\mathrm{Vycor}\right)$, they observed no change in the critical exponent from $\zeta \simeq 0.6705$ of bulk SF ${ }^{4} \mathrm{He}$, a fact clearly explained in terms of Harris criterion, provided that the pure critical system exhibits a negative specific heat exponent $\left(\alpha_{\text {pure }} \simeq-0.0105\right)$ [19]. Internal microchannel structures of Vycor, randomly oriented and randomly distributed, present scattering intensities that exhibit a peak near $r_{\max } \sim 20 \mathrm{~nm}$, and fall off exponentially above this peak, clearly faster than $r^{-d}$. In terms of the WH model, this ${ }^{4} \mathrm{He}-\mathrm{Vycor}$ system falls into the SRC regime (1), so theoretical predictions agree with experiments. Alternatively, Zassenhaus and Reppy [20], reported calorimetric studies for this ${ }^{4} \mathrm{He}-\mathrm{Vycor}$ system, 
showing that the singular part of specific heat curves fit well a $\lambda$-like curve with the same exponent $\alpha$ as bulk SF ${ }^{4} \mathrm{He}$. Further experiments, using porous gold to confine SF ${ }^{4} \mathrm{He}$, instead of Vycor, confirm these results [21]. Microchannels within these structures are similar to those of Vycor, but porosities are greater.

The challenge to theory comes in the case of ${ }^{4} \mathrm{He}$ confined in silica aerogels (AE) and xerogels $(\mathrm{XE})$, where authors observed $\zeta_{\mathrm{AE}} \simeq 0.81$ and $\zeta_{\mathrm{XE}} \simeq 0.89$, respectively, larger than the bulk exponent. After the WH model, these results suggest that LRC distributions of defects should be present within $\mathrm{AE}$ and XE. In practice, correlation functions are measured by means of (neutron/Xray) scattered intensities [12], thus algebraic (power-law) regimes on these plots correspond to algebraic regimes on $g(r)$. At these regimes, objects present self-similar (fractal) structure. Thus, provided that the condition (2) is fulfilled by the exponent $(a)$, critical exponents of the system will be modified by disorder.

Nevertheless, authors argue that silica AE certainly exhibit fractal regimes for several length scales, but they point out that beyond a finite cutoff $\Lambda_{\mathrm{AE}}$ curves enter homogeneous SRC regimes. Thus, close enough to the critical point, where the correlation length of SF ${ }^{4} \mathrm{He}$ diverges $\left(\xi \sim t^{-\nu}\right)$, disorder may appear homogeneous at the typical length scale of the system, and exponents should cross over to bulk values. For instance, in the case of $95 \%$ porous aerogels $\Lambda_{\mathrm{AE}} \simeq 150 \mathrm{~nm}$, and authors estimate $\xi(t) \simeq 480 \mathrm{~nm}$ already at $t \simeq 10^{-4}$, but although they run experiments up to $t \simeq 10^{-5}$, the crossover never appears [11]. They actually rule out an apparent violation to Harris criterion, but leave the open question about the LRC character of disorder, and propose the existence of LRC within aerogels, that cannot be observed through conventional techniques [7].

An attempt to explain these changes on the critical behaviour of $\mathrm{SF}^{4} \mathrm{He}$ will be discussed below, in section 3, showing that well defined LRC structures actually exist within aerogels. In the XE case, instead, a clear explanation to changes in critical exponents does not exist so far. As aerogels, these structures are created through silica sol-gel aggregation, but microstructures (strands) are broken and reorganized at the drying stage of the process, resulting in more compact (less porous) materials. It has been suggested [22] that, close to the flocculation-percolation transition in the aggregation process, strands present the same fractal dimension as the LRC structure, but still comparative studies do not reveal self-similarity in xerogels [23].

On the other hand, the liquid-vapor (LV) critical point, known to belong to the 3DIS universality class [24], gives another scope into the effect of aerogel-like disorder on phase transitions. In this case, any type of disorder, correlated or not, is relevant for criticality of the pure system. After Harris criterion [1], a positive specific heat exponent for the pure system $\left(\alpha_{3 \mathrm{DIS}} \simeq 0.109\right)$ makes random SRC disorder already relevant. In addition, theory predicts $[2,5]$ that the $O(1)$ universality class is also subject to changes in criticality under a LRC distribution of defects.

As we quote [13,17] and show below, both SRC and LRC structures are present within aerogels. Thus, the 3DIS confined in aerogel-like distributions of defects (AEIS) may be subject to these two competing effects. Results presented by Wong et al. [25], show that the LV critical point of fluid ${ }^{4} \mathrm{He}$ in aerogels exhibits specific heat curves with a finite peak at $T_{\mathrm{c}}$, which suggests that $\alpha_{\text {AEIS }}<0$, clearly different from the pure system exponent. In these and further experiments, using $\mathrm{N}_{2}$ instead [26], authors also report on the order parameter exponent concluding that, within error bars, it is indistinguishable from the pure system exponent. Theoretical predictions on the 3DIS with defects, give second order corrections to magnetic exponents with respect to those of the pure system. In addition, the coexistence curve is about 12 times narrower than that of the pure system [25], so it is not surprising that changes were so difficult to detect.

Then, the only trace left of an effect of aerogel-like disorder on the LV critical point is that concerning the finite peak of the specific heat. In a recent paper [27], Paredes and Vásquez show that this finite peak is consitent with a shift in the correlation length exponent to a value close to $2 / 3$, which is closer to the corresponding exponent for the randomly diluted Ising system (RDIS) [28-30], which is SRC at concentrations of disorder $c$ below the percolation critical value $p_{\mathrm{c}}$. However, it is hard to reach definitive asymptotic values for the exponents, due to an apparent oscillating approach to the fixed point. Thus the same question emerges from both experiments and simulations: why criticality would shift to the SRC and not to the LRC fixed point? 


\section{Correlations within aerogel-like distributions of disorder: DLCA}

In the sol-gel process of aerogel construction, silica dust is suspended in a solvent (sol phase), which allows diffusion and cluster-cluster aggregation to take place. Once the process has ended and the gel has been dried, detailed analysis of structure reveals that these objects are neither fractal nor LRC in the WH sense (2): only finite regions of power-law scaling are observed in scattering intensities from these objects [12]. However, at a given time $t_{\mathrm{g}}$ of the process, one of the clusters spans all the space of the flask, the gelling cluster (GC). Although it has not been tested experimentally, evidence exists that these GC are fractal at all scales [13] and LRC in the WH sense [17]. A numerical tool, the diffusion limited cluster-cluster aggregation algorithm (DLCA), developed in early 80's separately by Meakin [31] and Kolb, Botet, and Jullien [32], has proved to well reproduce the geometrical features of real aerogels [13].

As silica dust in suspension, in DLCA, particles that initially occupy random positions in a 3D box, are allowed to perform independent Brownian motions, and then keep together whenever two of them enter in contact. Undergoing this process, monomers and aggregates continue to move randomly, and attach themselves to each other until a unique cluster has been finally formed. In the on-lattice version of the algorithm, under periodic boundary conditions, $c L^{3}$ particles occupy random sites in a simple cubic $L^{3}$ box, being $c$ the concentration and $\varphi=1-c$ the porosity. A mass dependent diffusive constant is taken into account through a probability $p \sim m^{-\varsigma}$ for an aggregate of mass $m$ to move, where $\varsigma$ is a positive mobility exponent.

As in experiments, the GC is defined as the first cluster to reach opposites sides of the box in any direction. Right after the GC is built, many other smaller clusters (islands) continue to diffuse, and finally attach themselves at random sites to the GC. In the fabrication of an aerogel, the resulting structure is preserved by hypercritical evaporation of the solvent [12], so the GC matrix is kept almost intact, with randomly distributed strands attached to it. Islands represent the SRC subset within the whole cluster.

Figure 2 shows the geometrical features of different subsets of DLCA clusters, built in $L=128$ boxes at concentration $c=$ 0.05. Following a box-counting procedure [33], the scaling of corresponding average densities $n(r)=m(r) / r^{d}$ have been depicted as function of the box size $r$. The average over 32 realizations is taken. Plots marked as BBDLCA and BBGC correspond to backbones for the complete DLCA structure and the GC, respectively. Plots corresponding to complete DLCA clusters (circles) fast enter the homogeneous regime, due to islands attached to random sites at gelling clusters (squares): the slow powerlaw decay of GC alone suggest that these objects are LRC, but when islands have already reached the $\mathrm{GC}$ to finally form the whole DLCA structure, the LRC regime disappears screened (hidden) by the homogeneous distri-

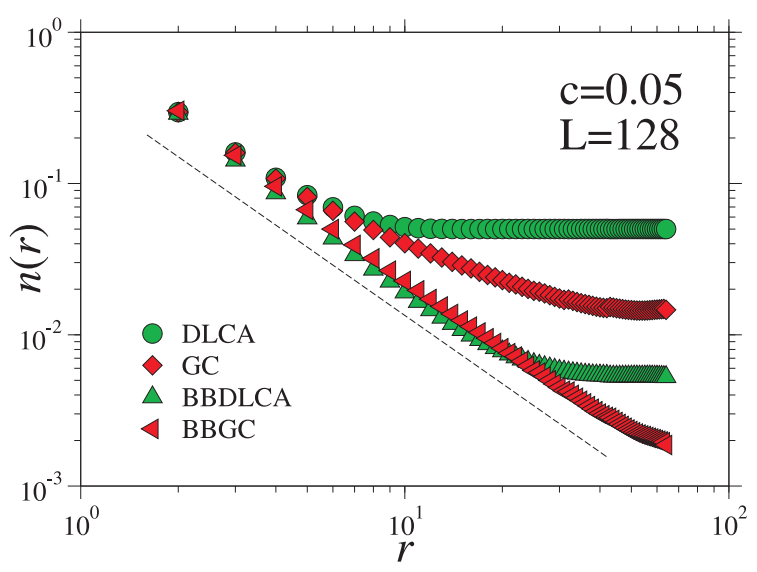

Figure 2. Average density $n(r)$ versus box size $r<L / 2$ for different subsets of $c=0.05$ DLCA clusters $(L=128)$. The dashed line represents the slope $a / 2=1.5$.

bution of islands. Backbones BBDLCA and BBGC also exhibit power-law long regimes, but as we argue just below, exponents larger than $3 / 2$ give $a>d=3$, which makes these subsets enter the SRC class, after WH criterion (1).

The average mass $m(r)$ scales as $r^{d_{\mathrm{f}}}$, being $d_{\mathrm{f}}$ the fractal dimension of the object. Recall that in the box-counting procedure one only takes non-empty boxes to estimate the average. This is equivalent to considering $n(r)$ as a one-point correlation function: considering only occupied sites as centers for $d$-dimensional cubes, the algorithm effectively measures the probability for surrounding sites to be occupied, normalized to $r^{d}$. This method rends a decaying exponent $\left(d-d_{\mathrm{f}}\right)$ for $n(r)$. We have argued [17] that the decaying exponent for this one-point correlation function is related 
to that of the impurity-impurity correlation function $g(r) \sim r^{-a}$ as $a=2\left(d-d_{\mathrm{f}}\right)$.

An example for this assertion comes from the percolation model, whose density-density correlation function scales as $g(r) \sim r^{-(d-2+\eta)}=r^{-a}$ at the critical occupation fraction $p=p_{\mathrm{c}}$. Using Rushbrooke hyperscaling relation one obtains $d-2+\eta=2 \beta / \nu$, being $\beta$ the critical exponent for the order parameter $\mathcal{P}_{\infty}$. Remind that the order parameter in the percolation problem is $\mathcal{P}_{\infty}=m_{\infty} / N$, where $m_{\infty}$ is the mass (size) of the percolating cluster at $p_{\mathrm{c}}$. This quantity scales as $P_{\infty} \sim L^{-\left(d-d_{\mathrm{f}}\right)}$, and being this the order parameter for the percolation transition [33], its exponent is defined as $d-d_{\mathrm{f}}=\beta / \nu$. In this case, it follows that the relation stated above, $a=2\left(d-d_{\mathrm{f}}\right)$ seems to hold [34].

For the complete DLCA cluster, the crossover to a homogeneous regime occurs at $r \ll L / 2$, while the fractal regime for backbones seems to span all scales. In the WH scheme, however, backbones present the fast decaying SRC regime (1), comparing the corresponding $n(r)$ with the slope 1.5 (dashed line). The long-range decay for the GC seems slower than $r^{-d}$, which enters the LRC regime (2). This case is more complex as it becomes homogeneous at $r \approx L / 2$, probably because some strands are already randomly attached to it at the gelation time $t_{\mathrm{g}}$. To show that GC are in effect fractal at the thermodynamical limit $L \rightarrow \infty$, while complete DLCA are not, plots of $n(r)$ for different lattice sizes $L=32-128$ are shown in figure 3. Arrows indicate crossover
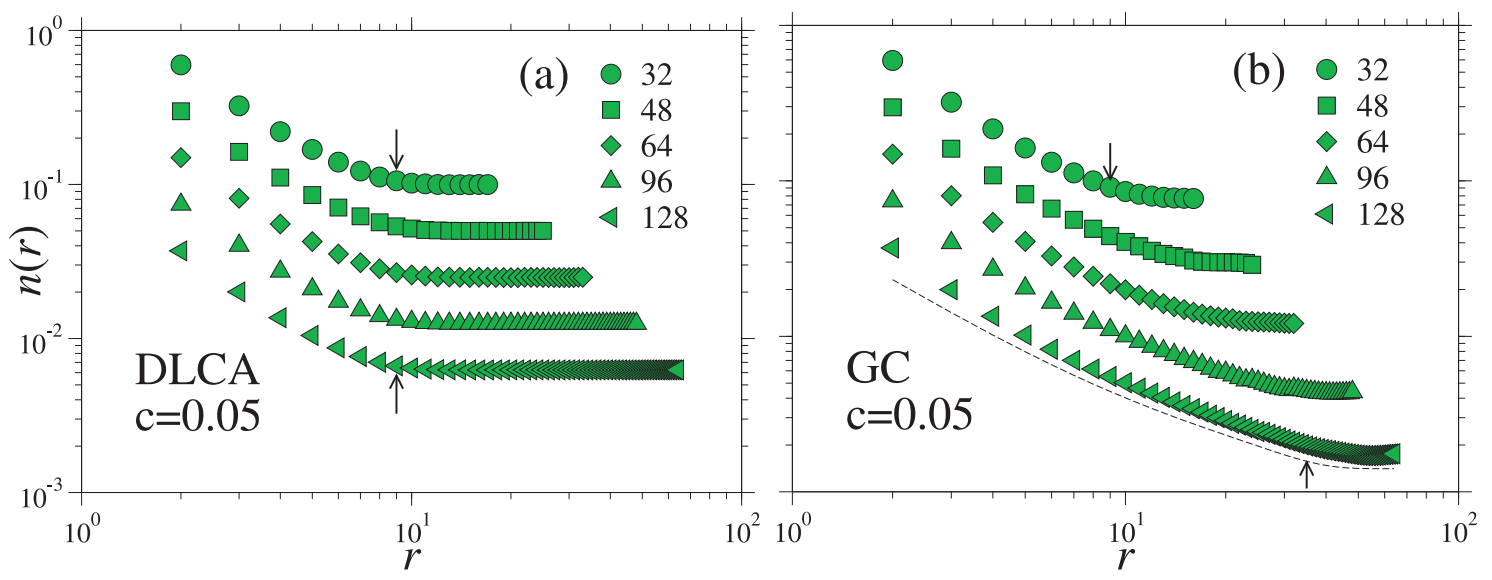

Figure 3. Average density $n(r)$ versus box size $r$ for (a) DLCA complete clusters and (b) corresponding GCs. Crossover to homogeneous regimes indicated by arrows. The dashed curve in (b) is the stretched exponential fit for the GC at $L=128$ (see text). Plots have been vertically displaced for better comprehension.

lengths which, for DLCA clusters (a), reach no more than a few lattice constants, independent of $L$. On the other hand, arrows in figure 3 (b) indicate that crossover lengths of GCs scale with the lattice size, a trace of fractality of these objects at $L \rightarrow \infty$. The complete DLCA cluster contains this LRC structure within, with numerous islands randomly attached to it, which hide correlations in the box-counting numerical method and make the whole structure appear homogeneous. This screening effect is unavoidable in experimental measurements of correlations (small-angle scattered intensities), resulting in finite fractal regimes for silica aerogels, which tend to disappear as density increases [12]. For simulated aerogels, finite size modifies the long-range behaviour of the correlation function. This effect is taken into account through the stretched exponential expression proposed by authors in $[22,35] n(r)=c_{\mathrm{g}}+A_{o} r^{-\left(d-d_{\mathrm{f}}\right)} \exp \left[-(r / \xi)^{\delta}\right]$, where $c_{\mathrm{g}}$ is the actual density of the GC (which tends to zero as $L \rightarrow \infty$ ) and $\xi$ is the characteristic radius of gyration (cuttoff). The parameter $\delta>1$ describes the faster crossover to the homogeneous regime characteristic of finite lattices. The dashed curve in figure 3 (b) represents the fit to this expression for $L=128$, giving the estimate $d_{\mathrm{f}} \approx 1.7$ (thus $a \approx 2.6$ ) for the fractal dimension of the GC, with a cutoff at $\xi \approx 35$. 


\section{4. $O(m)$-vector models with aerogel-like distribution of defects}

Monte Carlo simulations of $O(m)$-vector models consider the nearest neighbor interactions between spins, placed on a 3D simple cubic lattice of size $L\left(1<i<L^{3}\right)$, with periodical boundary conditions. When defects are present, these interactions are described by the Hamiltonian:

$$
\frac{\mathcal{H}}{k T}=-K \sum_{\langle i j\rangle} \epsilon_{i} \epsilon_{j} \vec{\phi}_{i} \cdot \vec{\phi}_{j}
$$

where $K=J / k T$ is the coupling (inverse temperature, with $k=J=1$ ), and $\epsilon_{i}=1$ if a spin occupies the site, or $\epsilon_{i}=0$ otherwise. Depending on the internal dimension $(m)$ of the order parameter, spins are taken as $\vec{\phi}_{i}= \pm 1$ for the 3DIS $(m=1)$, and $\vec{\phi}_{i}=\left(\cos \theta_{i}, \sin \theta_{i}\right)$ for the 3DXY $(m=2)$ model. For each independent disorder realization, an aerogel is first built by the DLCA on-lattice algorithm described above [32], taking each site from the complete final cluster as a defect. Aerogel pores, i. e., $N=(1-c) L^{3}$ empty sites left in the lattice, are then filled with spins, being $c$ is the concentration of defects. Monte Carlo sweeps (MCS) are then taken as follows, using Hamiltonian (3): Wolff cluster update algorithm [36] is implemented, taking one sweep as eight consecutive cluster flips, to reduce autocorrelations caused by critical slowing down. Here we quote the procedure followed in simulations of the 3DXY model [17]. For each disorder realization, thermalization is reached after $2 \times 10^{4}$ sweeps, while $2 \times 10^{6}$ production sweeps are taken at equilibrium for further statistical analysis. A suitable number of disorder realizations have been taken, depending on lattice sizes, which run from $L=16$ (256) to $L=64$ (16). The method is rather different in the disordered 3DIS [27], in that shorter time series $\left(\sim 10^{3} \mathrm{MCS}\right)$ are taken in statistics, increasing the number of realizations at each size $\left(\sim 2 \times 10^{3}\right)$. A detailed analysis on self-averaging of thermodynamical quantities, performed for the 3DIS case, shows a lack of selfaveraging in the magnetization, suceptibility and specific heat, and on the other hand, the energy is weakly self-averaged, with an exponent $x \approx 2.58$. In that work [27], disorder distributions of thermodynamic observables for simulations of the 3DIS in aerogels are shown to be more symetric and narrower than for RDIS. Thus, good rough estimates could be found with not so much disorder realizations for the aerogel case. For this reason, although such analysis has not been performed for results presented here for the 3DXY case, an acceptable agreement with experimental results on the SF transition of ${ }^{4} \mathrm{He}$ is obtained [17].

\subsection{Observables}

The magnetization squared (order parameter) is calculated as,

$$
M^{2}=\left[|\langle\vec{\phi}\rangle|^{2}\right]=\left[\left|\sum_{i} \vec{\phi}_{i} / N\right|^{2}\right],
$$

where brackets represent canonical ensemble averages, while square brackets express averages over disorder realizations, for fixed $L$ and $c$. The energy per spin is calculated as the canonical ensemble average:

$$
\mathcal{E}=[\langle E\rangle]=[\langle H\rangle / N]
$$

Moments for the energy per spin are estimated at the simulation coupling $K_{o}$, as $\mathcal{E}^{n}=\left[\left\langle E^{n}\right\rangle\right]$, as well as moments $M^{n}$ for the magnetization are estimated for the corresponding energy bins. Logarithmic derivatives of moments $n=1,2,4$ of magnetization are then calculated using the corresponding $E-M^{n}$ covariance in the canonical ensemble:

$$
\mathcal{D}_{n}=\frac{\partial \ln \left(M^{n}\right)}{\partial K}=-\frac{\left\langle M^{n} E\right\rangle-\langle E\rangle\left\langle M^{n}\right\rangle}{\left\langle M^{n}\right\rangle} .
$$

The specific heat is calculated through fluctuations of the energy per spin:

$$
\mathcal{C}=N K^{-2}\left(\left\langle E^{2}\right\rangle-\langle E\rangle^{2}\right) .
$$


The helicity modulus $\left[\left\langle\Upsilon_{\hat{\mu}}\right\rangle\right]$ has been determined using the Kubo formula [15,16], averaged over the three directions $\hat{\mu}=\hat{x}, \hat{y}, \hat{z}$, assuming that the model and disorder distributions are isotropic. This quantity is proportional to the superfluid density, as was derived by Fisher and colleagues [39], so it is directly related to the order parameter of the SF transition. We use this relation to show that the confined system (AEXY) acts in $d=3$ external dimension, $i$. e., aerogel pores are effectively three-dimensional [17].

These quantities have been stored from MC simulations at each realization of disorder and, after the application of reweighting techniques, extrapolation of thermodynamical quantities of interest are made at couplings $K \approx K_{o}[37,38]$.

\subsection{Finite size scaling: thermal exponents}

Maxima from reweighted curves for logarithmic derivatives of moments $n=1,2$, 4 of the magnetization (6), and the specific heat (7), as functions of the coupling $K$, are averaged over disorder to estimate thermal critical exponents.

\subsection{1. $O(2)$-vector models: $3 \mathrm{DXY}$ and AEXY}

According to the finite-size scaling (FSS) ansatz [40] maxima of logarithmic derivatives scale with lattice sizes $L$ as:

$$
\left[\mathcal{D}_{n}\right]_{\max } \sim L^{1 / \nu}
$$

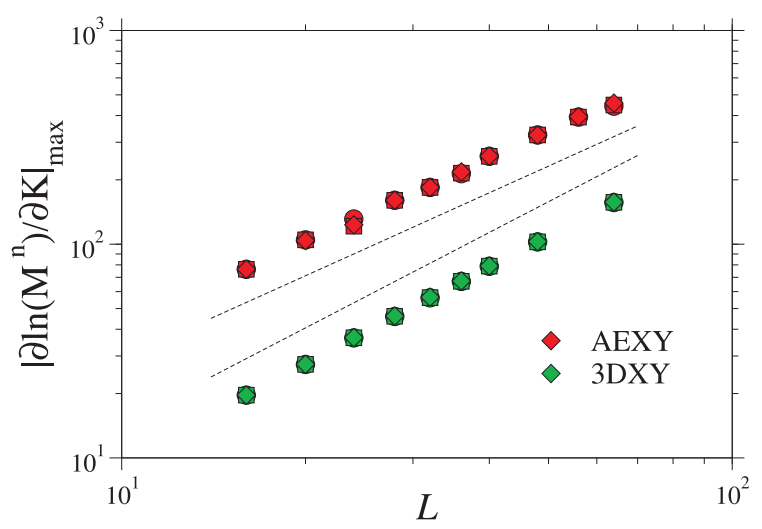

Figure 4. FSS of maxima of logarithmic derivatives $\mathcal{D}_{n}(n=1,2,4)$ for bulk $3 \mathrm{DXY}$ and the responding original plots have been vertically displaced to coincide at $L=16$. Dashed lines are fits to the FSS expression (8) on collected data. AEXY at concentration $c=0.05$ of defects. Cor-

Respectively, data for the 3DXY and the AEXY systems are depicted in figure 4, as function of lattice sizes $L$. Both groups of data have been vertically displaced (multiplied by a constant) to make corresponding plot coincide at $L=16$, which allows making a fit on collected data. The FSS power-law fit (8) for bulk 3DXY, using lattice sizes $L>24$ gives $1 / \nu_{\text {pure }}=1.483(3)$, which agrees well with previously reported results [41], and the exponent $\zeta \approx 0.67$ reported for bulk $\lambda$-transition [19]. The same fit to data for the AEXY, at concentration $c=0.05$, gives $1 / \nu_{\mathrm{AEXY}}=1.29(2)$, clearly different from the bulk value. Fits on figure 4 (dashed lines) have been vertically displaced to facilitate comparison between the slopes.

The correlation length exponent $\nu_{\mathrm{AEXY}} \approx$ 0.77 obtained for this confined system agrees well with the exponent $\zeta \approx 0.76$ obtained in experiments [9]. Both results, numerical and experimental, suggest that a LRC distribution of defects must be affecting the critical behaviour of the 3DXY universality class, when confined in aerogel-like structures. After the extended criterion (2), this structure should have a decaying exponent $a<2 / \nu_{\text {pure }} \approx 2.98$. Analysis on the DLCA structure resumed above gives $a \approx 2.6$ for gelling clusters of DLCA aerogels at $c=0.05$, so the WH condition is fulfilled and the distribution GC of disorder could be relevant. Nevertheless, there are other correlated structures within DLCA aerogels, the backbones, but given that $a>3$ for these structures, they do not satisfy the WH condition. If these structures have a decaying exponent smaller than $d$, figure 2 shows that this exponent is already greater than the corresponding exponent for the GC. The analysis resumed by WH [2] when multiple exponents are present on LRC strucutures, $i$. $e$., when $g(r)=\sum_{i} g_{i} r^{-a_{i}}$, it is the term with the smallest exponent which rules the critical behaviour for the impure system. Indeed, Prudnikov et al. [5] predict that for a decaying exponent similar to that reported above 
for the GC $(a \approx 2.6)$, the correlation length exponent should be $\nu_{\mathrm{LRC}} \approx 0.73$, so both experimental and numerical results roughly confirm this prediction. It is worth recalling that the whole DLCA structure itself is not LRC, but it is a mixture of SRC structures with a clearly LRC structure, the GC. As follows from WH results, SRC subsets are irrelevant. Thus, evident changes observed in critical exponents for this univerality class suggest that only the LRC subset rules the critical behaviour of both systems, SF ${ }^{4} \mathrm{He}$ and $3 \mathrm{DXY}$ interacting spins.

Moreover, we have further determined critical exponents for the specific heat and the helicity modulus as well. Maxima for the specific heat, assuming that $\alpha<0$, scale as:

$$
[\mathcal{C}]_{\max } \sim \mathcal{C}_{\infty}+A L^{\alpha / \nu}
$$

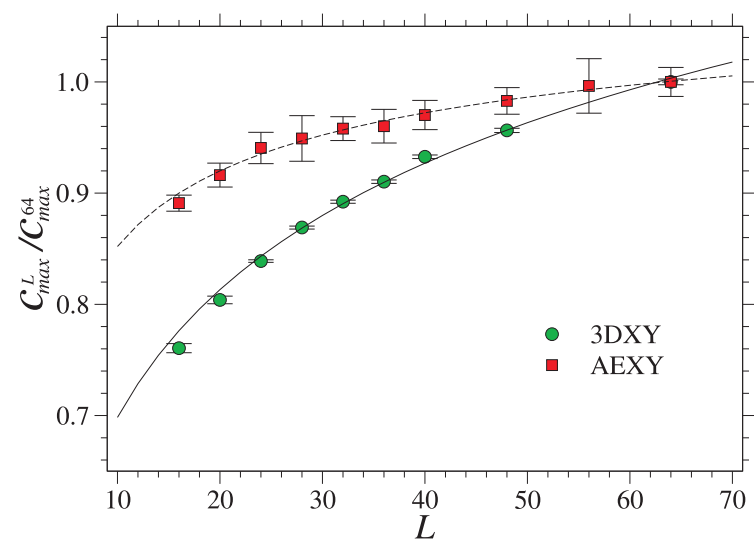

Figure 5. FSS of maxima of the specific heat for bulk 3DXY (circles) and confined AEXY at concentration $c=0.05$ of defects (squares). Lines are fits to the FSS expression (9). tain $\alpha \approx-0.010$ for bulk 3DXY, which agrees well with calorimetric studies on $\mathrm{SF}{ }^{4} \mathrm{He}$ [19]. The result $\alpha=-0.29$ for the AEXY is lower than the reported experimental value $\alpha-0.57$ for ${ }^{4} \mathrm{He}$ confined in aerogel pores at the same volume fraction $c=0.05$ [11]. However, in numerical simulations reported here, the question posed by authors about the apparent violation of hyperscaling seems not to emerge from our results on exponents $\nu$ and $\alpha$.

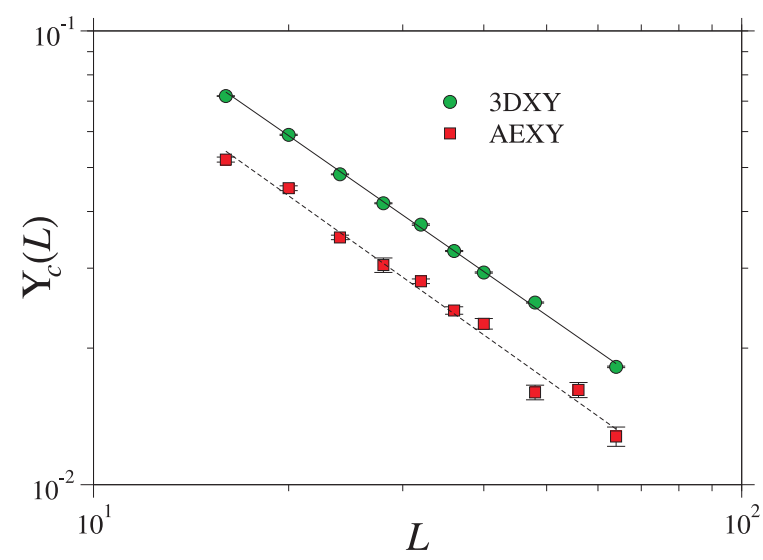

Figure 6. FSS plots of the helicity modulus at $K_{\mathrm{c}}$. Bulk 3DXY (circles) and confined AEXY at concentration $c=0.05$ of defects (squares).
These maxima have been depicted in figure 5 as function of $L$, normalized to $c(L=64) \equiv$ 1 , for bulk 3DXY (circles) and the confined AEXY system at $c=0.05$ (squares). Nonlinear fits of corresponding points to the FSS expression (9), using data for $L \geqslant 28$ to avoid corrections to scaling, are shown in figure 5 by corresponding lines. The method was developed by Schultka and Manousakis [42], to determine the specific heat exponent for the pure 3DXY system. The corresponding best fits give $\alpha / \nu \approx-0.015$ for the pure system, and $\alpha / \nu \approx-0.38$ for the confined system. These results agree well with our results on correlation length exponents, shown above, after the Josephson hyperscaling relation [18] in the form $2 / \nu-\alpha / \nu=d^{*}$, which gives $d^{*} \approx 3.0$ for both systems. Using corresponding results on the correlation length exponents, we obThe critical coupling $K_{\mathrm{c}}$ has been determined using the crossing of reweighted plots of the Binder fourth cumulant for the magnetization $U_{4}=1-\left\langle M^{4}\right\rangle / 3\left\langle M^{2}\right\rangle^{2}$ at different lattice sizes $L$, which is universal at $K_{\mathrm{c}}$ (independent of $L$ ) [43]. This analysis yields $K_{\mathrm{c}} \approx 0.45416$ for the pure system, while the crossing of average reweighted curves in the AEXY case gives $K_{\mathrm{c}} \approx 0.46495$.

Using these values, we estimate the helicity modulus at $K_{\mathrm{c}}$, averaged over disorder realizations:

$$
\left[\left\langle\Upsilon_{\hat{\mu}}\right\rangle\right] \sim L^{-v / \nu}
$$

Figure 6 shows corresponding data for the heand the AEXY case (squares). Lines represent fits to the FSS expression 10. The exponent licity modulus in the pure 3DXY case (circles), 
$v / \nu=1$ confirms that the effective external dimension for the system is $d=3$, through the hyperuniversality relation $v=(d-2) \nu$ [39]. This result also confirms that the correlation length exponent we have estimated here corresponds to the exponent $\zeta$ for the SF transition of ${ }^{4} \mathrm{He}$ in aerogels.

Results reported here seem to answer the question quoted above from [6,7]: numerical evidence has been presented here (section 3) that a physically well defined hidden LRC distribution of defects exists in aerogels, which explains changes on the critical behaviour of $\mathrm{SF}{ }^{4} \mathrm{He}$ when confined in those structures.

\subsection{2. $O(1)$-vector models: $3 \mathrm{DIS}$ and AEIS}

We turn now to a brief discussion on changes of the correlation length exponent for the 3DIS, when confined in DLCA aerogels at concentration $c=0.2$ of defects. A detailed analysis is reported elsewhere [27]. The method, as stated above, is quite different from that used in the AEXY case in that shorter MC time-series are used for extrapolation, but total numbers of realizations have been incremented in two orders of magnitude.

Figure 7. FSS plots of pseudocritical couplings $K_{\mathrm{c}}^{*}$ for the AEIS at $c=0.2$, taken from positions of maxima for the susceptibility $(\chi)$, and logarithmic derivatives $\left(\mathcal{D}_{n}\right)$. The horizontal axes have been rescaled to $L^{-1 / \nu}$, taking $1 / \nu=1.41$ (estimated in the insert).

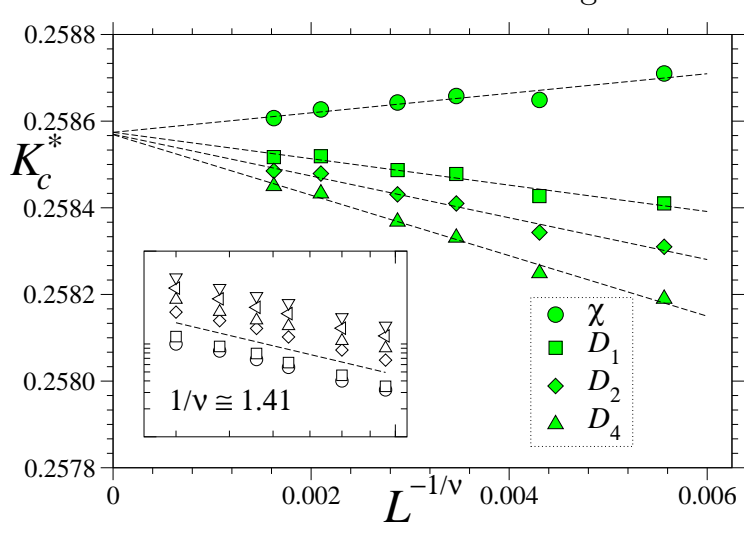

Shorter CPU times, in this case, permit to make simulations at greater lattice sizes, being $L=8-96$ in this case. After extrapolating by reweighting, curves have been averaged over disorder for each $K$, so a unique curve is obtained for each set of data. Position of maxima (pseudocritical couplings $K_{\mathrm{c}}^{*}(L)$ ) for the magnetic susceptibility $(\chi)$, and logarithmic derivatives $\mathcal{D}_{n}$ of moments of the magnetization $(n=1,2,4)$ have been estimated through the averaged curves. According to the finite size scaling theory [43], maxima for a given observable $\mathcal{O}$ scale as

$$
K_{\mathrm{c}}^{*}(\mathcal{O}, L)=K_{\mathrm{c}}+a(\mathcal{O}) L^{-1 / \nu}+\text { c. s. }
$$

$K_{\mathrm{c}}$ being the critical coupling at the thermodynamical limit, $L \rightarrow \infty$. Coefficients $a(\mathcal{O})$ are non-universal, $i$. e. , dependent on the observable $\mathcal{O}$ and on details of the system. Corrections to scaling (c. s. ) are avoided in the analysis reported below, taking data for large enough lattice sizes $(L>40)$. Points depicted on the insert of figure 7 , have been calculated through local differences $K_{\mathrm{c}}^{*}\left(\mathcal{O}_{i}, L\right)-K_{\mathrm{c}}^{*}\left(\mathcal{O}_{j}, L\right)$ between data from two different observables $\mathcal{O}_{i}$ and $\mathcal{O}_{j}$. This transformation of data permits to eliminate the non-singular term $\left(K_{\mathrm{c}}\right)$ from equation $(11)$, common by hypothesis to all observables, allowing for an easier estimation of the exponent on the leading term $a L^{-1 / \nu}$.

Power-law fits have been made to these transformed data, giving a rough estimate of the (common) FSS exponent $1 / \nu \approx 1.41$. This approximate has been used to rescale the horizontal axis to $L^{-1 / \nu}$, as indicated in figure 7 . As expected, a linear behaviour is observed for all data, and linear fits to the expression (11) give just one value $K_{\mathrm{c}}=0.25857(1)$ for all plots. A more detailed analysis on the scaling of logarithmic derivatives at the critical point reveals, however, that the correlation length exponent tends to a value closer to $3 / 2$ [27]. The correlation length exponent $\nu_{A E I S} \gtrsim 0.67$ obtained is clearly greater than that for the pure 3DIS and the LV critical point $(\nu \approx 0.63)$. These results point to a negative or zero value for the specific heat exponent $\alpha$. Experiments on the LV critical point of ${ }^{4} \mathrm{He}$ in aerogels [25] report specific heat curves that seem to have a finite peak at $T_{\mathrm{c}}$, but authors do not estimate the critical exponent $\alpha$ which may either be negative or zero as well. After Josephson hyperscaling, this is consistent with our rough result $1 / \nu \lesssim 1.5$, tending to values closer to that predicted for the SRC Ising universality class than that predicted for the LRC fixed point [5]. 
We should then conclude, in this case, that the FSS method applied here may not be precise enough to determine really asymptotic values for critical exponents, but a remarkable difference exists between the results approached here and the exponents for a pure system, as expected after Harris criterion.

\section{Concluding remarks}

Here we have reported on the effects of a specific type of disordered distributions of defects on the critical behaviour of $O(m)$-vector models, using the $O(1)$ and the $O(2)$ models, represented by the 3DIS and the 3DXY models, respectively. A detailed scaling analysis has been made to structures created through the DLCA on-lattice algorithm, which well reproduces the geometrical tasks of real silica aerogels. This analysis shows that the whole structure is not, strictly speaking, a fractal: the power-law decaying regime is preserved only through a few lattice constants, and even more, it does not scale with lattice sizes. A more detailed dissection shows the existence of physically well defined subsets within the whole strucuture, gelling clusters, that keep the algebraic decaying regime through lengths comparable to the lattice size, with a cutoff that scales with the system size. In the light of WH theory, the existence of this fractal (LRC) subset already explains the changes on critical exponents of the 3DXY model, when confined in these aerogel-like simulated structures, with respect to those of the pure system. Results for the confined (AEXY) system roughly confirm those observed for the SF transition of ${ }^{4} \mathrm{He}$ within aerogels, and explain why a crossover to bulk values is not observed in critical exponents reported from experiments, as $T \rightarrow T_{\mathrm{c}}$, when the correlation length diverges [11]. Even more intriguing are the results from the 3DIS confined in the same type of disorder distribution, as exponents seem to be affected by the LRC fixed point to finally tend values far from those predicted by theory [5], and closer to those of the SRC fixed point. The question about the critical behaviour of the AEIS rests open as conclusive results on asymptotic values for critical exponents have not yet been obtained. Previous renormalization group flows to fixed points, sketched by Weinrib and Halperin [2], and the analysis made by Prudnikov et al. [5], point out that the approach to the LRC fixed point may be oscillating, which can make it very dificult to enter a really asymptotic regime applying conventional finite size scaling.

\section{Acknowledgements}

Authors thank CNRS and FONACIT (PI2004000007) for their support. Invaluable discussions with A. Hasmy and R. Jullien have improved our understanding on aerogel structure. C. Vásquez kindly acknowledges the collaboration and technical support received by the personnel of the LCVN at Montpellier, France.

\section{References}

1. Harris A.B., J. Phys. C, 1974, 7, 1671; Harris A.B., Lubensky T.C., Phys. Rev. Lett., 1974, 33, 1540.

2. Weinrib A., Halperin B., Phys. Rev. B, 1983, 27, 207.

3. Aharony A., Harris A.B., Phys. Rev. Lett., 1996, 77, 3700.

4. Korshenevskii A.L., Herrmanns K., Schrimacher W., Phys. Rev. B, 1996, 53, 0144834.

5. Prudnikov V.V., Prudnikov P.V., Fedorenko A.A., Phys. Rev. B, 2000, 62, 8777.

6. Chan M.H.W., Blum K.I., Murphy S.Q., Wong G.K.S., Reppy J.D., Phys. Rev. Lett., 1988, 61, 1950.

7. Chan M.H.W., Mulders N., Reppy J.D., Phys. Today, 1996, 49 (8), 30.

8. Wong G.K.S., Crowell P.A., Cho H.A., Reppy J.D., Phys. Rev. Lett., 1990, 65, 2410.

9. Mulders N., Mehrotra R., Goldner L.S., Ahlers G., Phys. Rev. Lett., 1991, 67, 695.

10. Larson M., Mulders N., Ahlers G., Phys. Rev. Lett., 1992, 68, 3896.

11. Yoon J., Sergatskov D., Mulders J.Ma.N., Chan M.H.W., Phys. Rev. Lett., 1998, 80, 1461.

12. Vacher R., Woignier T., Pelous J., Phys. Rev. B, 1988, 37, 6500.

13. Hasmy A., Anglaret E., Foret M., Pelous J., Jullien R., Phys. Rev. B, 1994, 50, 6006; Hasmy A., Jullien R., J. Non-Cryst. Solids, 1995, 186, 342; Hasmy A., Jullien R., Phys. Rev. E, 1996, 53, 1789. 
14. Machta J., Phys. Rev. Lett., 1990, 66, 169.

15. Li Y.-H., Teitel S., Phys. Rev. B, 1990, 41, 11388.

16. Moon K., Girvin S.M., Phys. Rev. Lett., 1995, 75, 1328.

17. Vásquez C., Paredes R., Hasmy A., Jullien R., Phys. Rev. Lett., 2003, 90, 170602.

18. Josephson B.D., Phys. Lett., 1966, 21, 608.

19. Lipa J.A., Swanson D.R., Nissen J.A., Chui T.C.P., Israelsson U.E., Phys. Rev. Lett., 1996, 76, 944; Lipa J.A., Nissen J.A., Stricker D.A., Swanson D.R., Chui T.C.P., Phys. Rev. B, 2003, 68, 174518.

20. Zassenhaus G.M., Reppy J.D., Phys. Rev. Lett., 1999, 83, 4800.

21. Yoon J., Chan M.H.W., Phys. Rev. Lett., 1997, 78, 4801.

22. Gimel J.Ch., Durand D., Nicolai T., Phys. Rev. B, 1995, 51, 11348.

23. Lattuada M., Wu H., Morbidelli M., Phys. Rev. E, 2001, 64, 61404.

24. Pestak M.W., Chan M.H.W., Phys. Rev. B, 1984, 30, 274.

25. Wong A.P.Y., Chan M.H.W., Phys. Rev. Lett., 1990, 65, 2567.

26. Wong A.P.Y., Kim S.B., Goldburg W.I., Chan M.H.W., Phys. Rev. Lett., 1993, 70, 954.

27. Paredes R., Vásquez C. Preprint arXiv: cond-mat/0604592.

28. Folk R., Holovatch Yu., Yavors'kii T., Phys. Rev. B, 2000, 61, 15114; Holovatch Yu., Yavorskii T., J. Stat. Phys., 1998, 92, 785.

29. Calabrese P., Martín-Mayor V., Pelissetto A., Vicari E., Phys. Rev. E, 2003, 68, 036136.

30. Pelissetto A., Vicari E., Phys. Rev. B, 2000, 62, 6393.

31. Meakin P., Phys. Rev. Lett., 1983, 51, 1119.

32. Kolb M., Botet R., Jullien R., Phys. Rev. Lett., 1983, 51, 1123; J. Phys. A, 1983, 17, L75.

33. Feder J. Fractals, 1988, Plenum Press, New York.

34. This is not, of course, a rigurous mathematical prove. Authors in [2,5] define $g(r)$ as the correlation function for the local critical temperature $g\left(\left|\overrightarrow{\boldsymbol{r}}_{1}-\overrightarrow{\boldsymbol{r}_{2}}\right|\right)=\left\langle T_{\mathrm{c}}\left(\overrightarrow{\boldsymbol{r}}_{1}\right) T_{\mathrm{c}}\left(\overrightarrow{\boldsymbol{r}}_{2}\right)\right\rangle \sim\left|\overrightarrow{\boldsymbol{r}}_{1}-\overrightarrow{\boldsymbol{r}_{2}}\right|^{-a}$. We relate fluctuations in this local $T_{\mathrm{c}}(\overrightarrow{\boldsymbol{r}})$ with the local density of surrounding defects $n(\overrightarrow{\boldsymbol{r}})$, and it is in terms of this density function that we made approximate calculations about $g(r)$, which allow relating the exponent $a$ to the fractal dimension $d_{\mathrm{f}}$ of the distribution.

35. Lach-hab M., González A.E., Blaisten-Barojas E., Phys. Rev. E, 1998, 57, 4520.

36. Wolff U., Phys. Rev. Lett., 1989, 62, 361.

37. Ferrenberg A.M., Swendsen R.H., Phys. Rev. Lett., 1988, 61, 2635; Ferrenberg A.M., Landau D.P., Phys. Rev. B, 1991, 44, 5081.

38. Newman M.E.J., Barkema G. Monte Carlo Methods in Statistical Physics. Oxford University Press, New York, 2000.

39. Fisher M.E., Barber M.N., Jasnow D., Phys. Rev. A, 1973, 8, 1111.

40. Fisher M.E., Barber M.N., Phys. Rev. Lett., 1972 28, 1516; Fisher M.E., Rev. Mod. Phys., 1972, 46, 597.

41. Hasenbusch M., Török T., J. Phys. A, 1999, 32, 6361.

42. Schultka N., Manousakis E., Phys. Rev. B, 1995, 52, 7528.

43. Binder K., Phys. Rev. Lett., 1981, 47, 693. 


\section{Questions and answers}

$\mathcal{Q}$ (Rafael Rangel): What are the theoretical predictions for the (possible) change of exponents by disorder?

$\mathcal{A}$ Harris criterion predicts that a weak uncorrelated disorder is not relevant if the correlation length exponent $\nu$ is larger than $2 / d$ for the pure system, or after Josephson hyperscaling, $\alpha=2-d \nu<0$. For 3DIS case, $\alpha>0$, and then short-range-correlated disorder could be relevant. Weinrib and Halperin extend this criterion to cases where there exist long-range correlations (LRC) in the distribution of defects, as expressed by equations (1) and (2). It is this extension that permits to explain explain the changes on the critical behaviour of the 3DXY model when confined in aerogel-like distributions of defects, and the changes on critical exponents of the SF transition of ${ }^{4} \mathrm{He}$ in aerogels as well.

$\mathcal{Q}$ (Dragi Karevski): How does your FSS technique work if there are different correlations?

$\mathcal{A}$ Weinrib and Halperin [2] have already considered the case when there exist terms with different decaying exponents in the correlation function $g(r)$, as was quoted in Subsection 4.2.1: the critical behaviour couples to the term with the lowest exponent. Indeed, in the model disorder we are considering in simulations presented here, DLCA, there exist several subsets of the whole cluster which present different decaying regimes. Results point to state that, in the 3DXY confined case (AEXY), it is the gelling cluster (GC) that rules the critical behaviour, as it is the LRC subset with the lowest exponent (see figure 2). The AEIS case is by far more interesting in that competing effects are present, as SRC disorder is also relevant. Being the LRC fixed point marginal, it seems that it is the SRC fixed point that finally governs the critical behaviour, $i$. e., the effective exponent $1 / \nu$ turns from being attracted to $1 / \nu \approx 1.4$ (predicted by Prudnikov et al. [5] for the LRC fixed point) to $1 / \nu \lesssim 3 / 2$, closer to the SRC fixed point value. Nevertheless, even if only one model LRC distribution of defects were present, the application of FSS techniques should consider that the convergence of the correlation function to the final decaying regime $L^{-\left(d-d_{\mathrm{f}}\right)}$ will depend on the lattice size $L$, so the convergence of effective exponents to critical values will be ruled by an exponent that asymptotically tends to a stable value. This task of the correlation function may cause succesive crossover regimes which appear in the convergence of effective exponents to asymptotic critical exponents.

\section{$\mathcal{Q}$ (Dragi Karevski): How does the FSS with $\boldsymbol{V}=\boldsymbol{L}^{\boldsymbol{d}}$ (and not with $\boldsymbol{L}$ ) hold?}

$\mathcal{A}$ The FSS theory is based on the homogeneity of the free energy density, which permits to write it in terms of the scaling variable $L / \xi=L t^{\nu}$, which at its time may be redimensioned to $t L^{-1 / \nu}$. The external dimension $d$ of the system appears only in corresponding hyperscaling relations, such as those of Josephson $2 / \nu-\alpha / \nu=d$ and Rushbrooke $\gamma / \nu+2 \beta / \nu=d$.

\section{$\mathcal{Q}$ (Wolfhard Janke): What is the dimension $\boldsymbol{d}_{\mathbf{f}}$ of your fractal?}

$\mathcal{A}$ The whole DLCA clusters in our model disorder are not fractal, as they enter homogeneous regime at very few lattice constants, but the well defined GC have correlations that seem to span all the scales at thermodynamical limit. The best estimate we have of the fractal dimension for these objects is $d_{\mathrm{f}} \simeq 1.7$.

\section{$\mathcal{Q}$ (Yurij Holovatch): How is $\boldsymbol{c}$ related to $\boldsymbol{a}$ ?}

$\mathcal{A}$ As the concentration of defects increases, the fractal dimension of the GC increases as well, as stated in [13], so the exponent $a$ decreases. 


\title{
Вплив безладу типу аерогелю на критичну поведінку $O(m)$-векторних моделей. Недавні симуляції та експериментальні прояви
}

\author{
К.Васкес ${ }^{1}$, Р.Паредес ${ }^{2,3}$ \\ 1 Фізичний факультет, Університет Сімона Болівара, \\ пошт. скринька 89000, Каракас 1080-А, Венесуела \\ 2 Центр фізики, Венесуельський інститут наукових досліджень, \\ пошт. скринька 21827, Каракас 1020-А, Венесуела \\ 3 Група технології частинок, Технологічний університет, \\ Дельфт, 2629 BL, Нідерланди
}

Отримано 10 квітня 2006 р., в остаточному вигляді - 12 травня 2006 р.

Проведено огляд недавніх результатів дослідження впливу певного типу замороженого безладу на добре відомі $O(m)$-векторні моделі у трьох вимірах: $X Y$ модель (3DXY, $m=2$ ) і модель Ізинга (3DIS, $m=1$ ). Коротко згадуються прояви змін в критичності обох систем у порах аерогелів. 3DXY модель $€$ представником класу універсальності, до якого належить $\lambda$-перехід надплинного ${ }^{4} \mathrm{He}$. Експерименти свідчать про цікаві зміни в критичних показниках цього переходу, коли надплинний ${ }^{4}$ Не міститься в аерогелях. Були зроблені також часові симуляції, які підтверджують те, що 3DXY модель на гратці із структурою дефектів, подібних до структури аерогелю, характеризується критичними показниками, відмінними від чистого випадку. На перший погляд видається, що обидва результати суперечать критерію Гарріса. Але у випадку, коли критичний показник питомої теплоємності чистої системи від'ємний, $\left(\alpha_{3 \mathrm{DXY}} \simeq-0.011<0\right)$, зміни повинні пояснюватися узагальненим критерієм Вайнріба та Галперіна, який стосується далекосяжно-скорельованого на всіх масштабах безладу. У чисельних симуляціях аерогелі моделюються алгоритмом керованої дифузією кластер-кластерної агрегації. Відомо, що такий алгоритм відтворює певні геометричні характеристики аерогелів. Ці об'єкти, чи реальні, чи симульовані, $€$ фракталами лише на певних масштабах. Із збільшенням масштабу відбувається перехід до однорідного режиму. В свою чергу, це спричиняє порушення критерію Гарріса. Таке порушення було пояснене присутністю в аерогелях прихованих далекосяжно-скорельованих включень [Phys. Rev. Lett., 2003, 90, 170602]. 3 іншого боку, підчас експериментальних досліджень переходу рідина-пара в ${ }^{4} \mathrm{He}$ і $\mathrm{N}_{2}$ в аерогелях також спостерігалась зміна показників у критичній точці. Якби цей перехід був у класі універсальності $O(1)$, то на критичну поведінку впливав би і короткосяжно-, і далекосяжно-скорельований безлад. Симуляції 3DIS в аерогелях, модельованих алгоритмом корельованої дифузією кластер-кластерної агрегації, підтверджують експериментальні результати. I експерименти, і симуляції свідчать про те, що значення критичних показників стають ближчими до значень у короткосяжно-скорельованій, а не в далекосяжно-скорельованій нерухомій точці.

Ключові слова: фазові переходи, векторні моделі, скорельований безлад, аерогелі

PACS: $64.60 . \mathrm{Cn}, 64.60 . \mathrm{Fr}, 64.70 .-p$ 
